\title{
Integrating Spline Curves in Road Constraint Object Tracking
}

\author{
Carsten Hasberg, Stefan Hensel, Maximilian \\ Westenkirchner, Karolina Bach \\ Institut für Mess- und Regelungstechnik \\ Universität Karlsruhe \\ 76131 Karlsruhe, Germany \\ hasbergamrt.uka.de
}

\begin{abstract}
Including road map information in the tracking of ground moving objects is a challenging problem. While many self localizing algorithms base on a modelling in global Cartesian coordinates a few approaches prefer a modelling in a local map coordinate frame. Throughout this contribution both strategies are compared theoretically and in numerical simulations. To achieve robust tracking results current onboard sensor information is utilized in the tracking framework. Commonly available on cars or trains are absolute position information, estimated e.g. by a GPS unit, and relative velocity measurements, e.g. measured by an odometer. To integrate road map knowledge with this sensor information time- and measurement-update equations are derived for both modelling strategies.

Roads or tracks are often composed by a sequence of geometric primitives. Approximating this progression of geometric elements with smooth piecewise defined polynomials yields an accurate model, which can easily be integrated in the tracking framework. General preconditions using curves for tracking purposes are presented. In particular, cubic Hermite spline curves are chosen and implemented into the tracking framework.
\end{abstract}

\section{INTRODUCTION}

Incorporating prior information about dynamic behaviour and measurement model both have a strong impact on the performance of object tracking algorithms [1]. For ground moving objects assumptions on road- or track-constrained motion may augment significant information to tracking processes. To obtain robust object tracking results, there exist several concepts to include road map information into the tracking algorithm [2] [3].

To perform a robust road constraint object tracking an adequate approximation of the road network is to be realized initially. Roads or tracks are often composed by a sequence of geometric primitives, e.g. straights, transition curves and circular arcs, to enable a comfortable and save driving without any abrupt variations in lateral acceleration. A prominent way to store this geometric knowledge are piecewise defined linear polynomial models [3] [4]. If additional information about road tangents is available a more precise geometric approximation can be achieved, making use of higher order polynomials. In this paper cubic Hermite splines are selected. One characteristic of these curves is their locality: If the road geometry changes in a defined section and the road map has to be updated, only a limited modification of the geometric parameters has to be executed.
Furthermore, in many applications the only point of interest concerning these polynomial curves is their interpolation error, while the type of parameterization is of less interest. By contrast, realizing object tracking in local curve coordinates requires not only a correct geometric run of the curve but although a correct arc-length parameterization. Therefore a method is proposed to achieve a sufficient approximation of arc-length parameterization, while the numerical effort remains manageable.

Given a geometric description of the road constraints, a mathematical formulation of the estimation problem has to be chosen, combining this road map knowledge with current sensor measurements. Because commonly available, absolute position and relative velocity measurements are chosen to support the object tracking. Two classes of concepts are being compared:

In many applications the movement of objects is modelled in Cartesian coordinates [2]. In the two or three dimensional case the movements of each component are often assumed to be decoupled. In case of curved roads the position of the object then leaves the road constraint during each time- and measurement-update step. One opportunity to include road map information can then be realized in a post filter processing step - often called map matching [5]. Alternatively, constrained Kalman filter techniques systematically include road map knowledge [4], but linearization errors, that occur during the time update, remain.

The second class of concepts formulates the dynamic behaviour in local curve coordinates [3] [6]. If the representation of the road network fulfils several preconditions this strategy yields in robust results. The road map knowledge is included effectively and a significant improvement of the object tracking accuracy can be observed compared to the global modelling.

Throughout this contribution both concepts are going to be compared theoretically and in numerical simulations. Preconditions for both approaches are analyzed and object tracking results presented. In comparison with former approaches the geometric properties of the track- or road constraints are modelled more precisely. Therefore smooth piecewise defined third order polynomials are used to achieve minimal interpolation errors, especially in case of strongly curved roads. In addition, a simple but sufficient method to 
approximate arc-length parameterization is proposed. Both modelling strategies result in nonlinear state-to-measurement relations. To update the state estimation an extended Kalman filter is implemented.

This paper is organized as follows: In Section II the available map information source is presented. To combine these road map database with additional sensor information principles of information fusion are expressed in Section III. Both strategies to model objects moving along roads are presented in Section IV. Finally the concepts are compared in numerical simulations in Section $\mathrm{V}$ and a conclusion summarizes the results in Section VI.

\section{ROAD MAP}

The road network is interpolated with naturally parameterized piecewise defined curves. To achieve a smooth and precise approximation of the road, cubic Hermite spline curves are chosen.

Each element of the road network is given by a set of absolute positions $\mathbf{p}_{i}$ and tangents $\tau_{i}$ for each of the $n$ supporting points. First step is to determine parameter values for each vertex $\mathbf{p}_{i}$. Chord length parameterization [7] is chosen to achieve a parameterization proportional to the distances of the data points and to approximate arclength parameterization. The curve parameter values $u_{i}$ for $\mathbf{p}_{i}=\mathbf{p}_{i}\left(u_{i}\right)$ are calculated according to

$$
u_{i+1}=u_{i}+\left\|\mathbf{p}_{i+1}-\mathbf{p}_{i}\right\|
$$

as sketched out in Figure 1. The initial chord length is set to $u_{0}=0$.

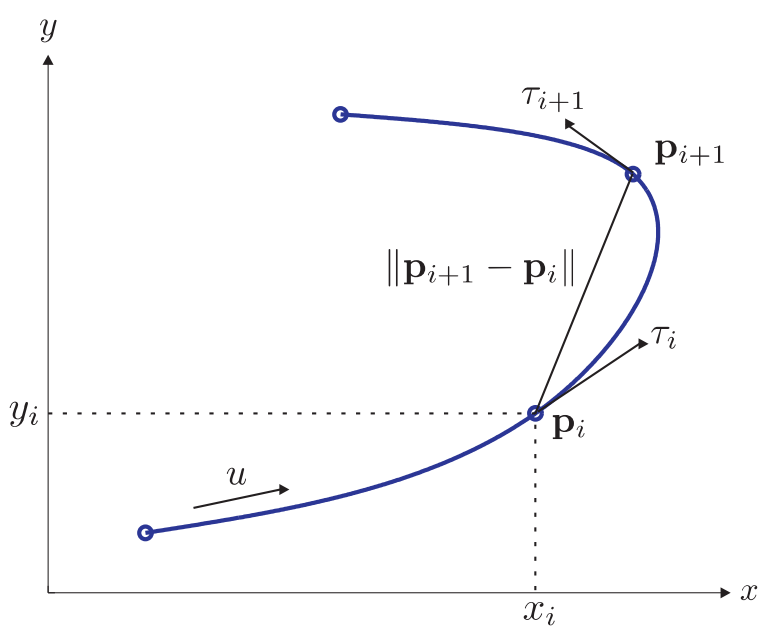

Fig. 1. Parameterization of curve element

While data points $\mathbf{p}_{i}$, tangents $\tau_{i}$ and parameter values $u_{i}$ are available, both coordinate functions are interpolated separately with splines. For $u \in\left[u_{i}, u_{i+1}\right]$ the coordinate functions are defined piecewise with

$$
\mathbf{s}(u)=\left(\begin{array}{c}
s_{x}(u) \\
s_{y}(u)
\end{array}\right)=\left(\begin{array}{c}
s_{x}^{(i)}(u) \\
s_{y}^{(i)}(u)
\end{array}\right)=\mathbf{s}^{(i)}(u)
$$

with

$$
\begin{aligned}
s_{x}^{(i)}(u) & =a_{x, i}+b_{x, i} \Delta u_{i}+c_{x, i} \Delta u_{i}^{2}+d_{x, i} \Delta u_{i}^{3} \\
s_{y}^{(i)}(u) & =a_{y, i}+b_{y, i} \Delta u_{i}+c_{y, i} \Delta u_{i}^{2}+d_{y, i} \Delta u_{i}^{3}
\end{aligned}
$$

and $\Delta u_{i}=u-u_{i}$.

To calculate the geometric curve parameters $a_{x, i}, \ldots, d_{y, i}$, a set of smoothness conditions has to be fulfilled. These conditions are

$$
\begin{aligned}
\mathbf{s}^{(i)}\left(u_{i}\right)=\mathbf{p}_{i} & \mathbf{s}^{(i)}\left(u_{i+1}\right)=\mathbf{p}_{i+1} \\
\dot{\mathbf{s}}^{(i)}\left(u_{i}\right)=\tau_{i} & \dot{\mathbf{s}}^{(i)}\left(u_{i+1}\right)=\tau_{i+1}
\end{aligned}
$$

for $i=0, \ldots, n-1$.

Solving the resulting system of equations for each element of the piecewise defined spline curve gives

$$
\mathbf{s}(u)=\mathbf{B}^{T}(u)\left(\begin{array}{c}
\mathbf{p}_{i} \\
\mathbf{p}_{i+1} \\
\tau_{i} \\
\tau_{i+1}
\end{array}\right)
$$

for $u \in\left[u_{i}, u_{i+1}\right]$ with

$$
\mathbf{B}=\left(\begin{array}{c}
2\left(u_{i+1}-u_{i}\right)^{-3} \Delta u_{i}^{3}-3\left(u_{i+1}-u_{i}\right)^{-2} \Delta u_{i}^{2}+1 \\
-2\left(u_{i+1}-u_{i}\right)^{-3} \Delta u_{i}^{3}+3\left(u_{i+1}-u_{i}\right)^{-2} \Delta u_{i}^{2} \\
\left(u_{i+1}-u_{i}\right)^{-2} \Delta u_{i}^{3}-2\left(u_{i+1}-u_{i}\right)^{-1} \Delta u_{i}^{2}+\Delta u_{i} \\
\left(u_{i+1}-u_{i}\right)^{-2} \Delta u_{i}^{3}-\left(u_{i+1}-u_{i}\right)^{-1} \Delta u_{i}^{2}
\end{array}\right)
$$

The matrix entries of $\mathbf{B}(u)$ are often called basis functions of the spline. For further details see [7].

The resulting curve is given in cord length parameterization and the parameterization error $\epsilon(u)=l(u)-u$ increases along the arc-length

$$
l(u)=\int_{0}^{u}\|\dot{\mathbf{s}}(u)\| d u
$$

of the spline. Therefore re-parameterization is necessary to achieve arc-length or natural parameterization [8].

Here a simple method to approximate arc-length parameterization is proposed, that yields a bias free error $\epsilon(u)$, while the calculation effort remains small. Based on the current geometric run of the spline curve $\mathbf{s}(u)$ the exact arc-length is calculated for each vertex according to

$$
l_{i+1}=l_{i}+\int_{u_{i}}^{u_{i+1}}\|\dot{\mathbf{s}}(u)\| d u .
$$

Finally each $u_{i}$ is replaced by the corresponding $l_{i}$.

For a simple example curve (length approximately $120 \mathrm{~m}$ ) starting with a radius of $25 \mathrm{~m}$ and ending in a straight line the modification strategy offers quite good results as depicted in Figure 2. After an initial approximation with cord-length parameterization the error $\epsilon_{1}(u)$ rises on. Improving the parameterization with the proposed algorithm results in a bias free error $\epsilon_{2}(u)$. 


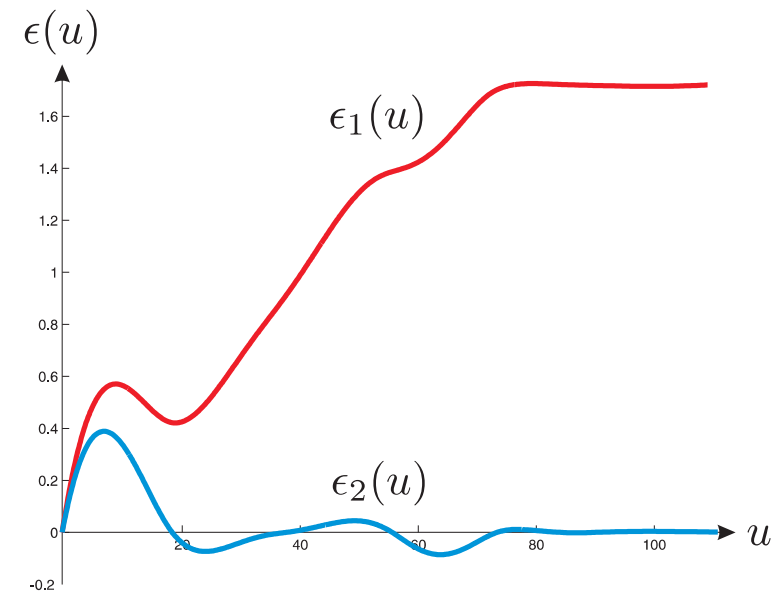

Fig. 2. Parameterization error function in (m) along the example curve for cord length parameterization $\epsilon_{1}(u)$ and after proposed arc-length parameterization $\epsilon_{2}(u)$

\section{STATE ESTIMATION WITH THE EXTENDED KALMAN FILTER}

Throughout this contribution the index $(\ldots)^{-}$is used for the estimation of the state vector before the measurement update and the index $(\ldots)^{+}$for the estimation after the update.

The extended Kalman filter [1] [9] is a tool to estimate a state vector that can be observed through indirect measurements which are subject to noise. The functional dependency between a state vector $\mathbf{x}_{k}$ and the measurement vector $\tilde{\mathbf{z}}_{k}$ is modelled by the matrix equation

$$
\tilde{\mathbf{z}}_{k}=h\left(\mathbf{x}_{k}\right)+\mathbf{w}_{k} .
$$

The measurement noise $\mathbf{w}_{k}$ is assumed white and zero-mean. Its covariance matrix $\mathbf{R}$ describes all deviations from the expected behaviour.

The extended Kalman filter allows incorporating knowledge of system dynamics into the estimation modelled by the matrix equation

$$
\mathbf{x}_{k+1}=\mathbf{f}\left(\mathbf{x}_{k}\right)+\mathbf{v}_{k}
$$

predicting the state vector from one discrete time instant $k$ to the next. The system noise $\mathbf{v}_{k}$ with the covariance matrix $\mathbf{Q}$, is again assumed to be white, zero-mean.

If both error sources can be assumed to be additive and mutual statistically independent the resulting state estimation minimizes the mean square error. In case of nonlinear equations the state vector $\mathbf{x}$ is estimated approximately biasfree with mean vector $\hat{\mathbf{x}}$ and with covariance matrix $\mathbf{P}$.

The Kalman filter state estimation is computed in two main steps [10]. Initially the estimate of the state vector is predicted with

$$
\begin{aligned}
\hat{\mathbf{x}}_{k} & =\mathbf{f}\left(\hat{\mathbf{x}}_{k-1}\right) \\
\mathbf{P}_{k} & =\mathbf{F}_{k-1} \mathbf{P}_{k-1} \mathbf{F}_{k-1}^{T}+\mathbf{Q}
\end{aligned}
$$

with $\mathbf{F}_{k-1}$ is the Jacobian matrix calculated at the current state estimation according to

$$
\mathbf{F}_{k-1}=\mathbf{f}^{\prime}\left(\hat{\mathbf{x}}_{k-1}\right) \text {. }
$$

The measurement update equations

$$
\begin{aligned}
\mathbf{K}_{k} & =\mathbf{P}_{k}^{-} \mathbf{H}_{k}^{T}\left(\mathbf{H}_{k} \mathbf{P}_{k}^{-} \mathbf{H}_{k}^{T}+\mathbf{R}\right)^{-1} \\
\hat{\mathbf{x}}_{k}^{+} & =\hat{\mathbf{x}}_{k}^{-}+\mathbf{K}_{k}\left(\tilde{\mathbf{z}}_{k}-h\left(\hat{\mathbf{x}}_{k}^{-}\right)\right) \\
\mathbf{P}_{k}^{+} & =\left(\mathbf{I}-\mathbf{K}_{k} \mathbf{H}_{k}\right) \mathbf{P}_{k}^{-}
\end{aligned}
$$

adjust the predicted estimation with new incoming measurement information. Again $\mathbf{H}_{k}$ is the Jacobian matrix calculated with

$$
\mathbf{H}_{k}=\mathbf{h}^{\prime}\left(\hat{\mathbf{x}}_{k}\right) \text {. }
$$

\section{OBJECTS ON ROADS}

Observing an object with variable position $x$, idealized moving with constant velocity $v_{x}$, there are a couple of ways to model uncertainty. To explain variations in velocity, state of the art algorithms base on the assumption of white acceleration [1].

To track objects moving in two or three dimensions often an identical model is used for each coordinate, while the movements along each coordinate direction are assumed to be uncorrelated from the others.

A prominent way to describe the object tracking task is a formulation of the concept in global Cartesian coordinates. If a road map is given, a formulation in local curve coordinates can be used alternatively. Throughout this chapter, timeand measurement update equations for both concepts are presented to realize an object tracking with the extended Kalman filter as described in Section III. The need of a map matching step is discussed for both formulations.

For the sake of clarity, the whole concept is described for planar case. However, it easily generalizes to $3 \mathrm{~d}$-description.

\section{A. Model in Global Coordinates}

Assuming linear motion in $x y$-coordinate frame the evolution of the object state $\mathbf{x}_{k}=\left(x_{k} v_{x, k} y_{k} v_{y, k}\right)^{T}$ can be written as

$$
\mathbf{x}_{k+1}=\mathbf{F} \mathbf{x}_{k}+\mathbf{G} a_{k}
$$

where $a_{k}$ is white Gaussian process noise with variance $\sigma_{a}^{2}$. It is

$$
\mathbf{F}=\left(\begin{array}{cccc}
1 & T & 0 & 0 \\
0 & 1 & 0 & 0 \\
0 & 0 & 1 & T \\
0 & 0 & 0 & 1
\end{array}\right) \text { and } \mathbf{G}=\left(\begin{array}{cc}
\frac{T^{2}}{2} & 0 \\
T & 0 \\
0 & T \\
0 & \frac{T^{2}}{2}
\end{array}\right)
$$

where $T$ is the sampling interval.

Caused by the linear state-to-measurement relationship of position measurements $\tilde{\mathbf{z}}_{p o s, k}=\left(\tilde{x}_{k} \tilde{y}_{k}\right)^{T}$ the measurement equation can be written as

$$
\tilde{\mathbf{z}}_{p o s, k}=\left(\begin{array}{cccc}
1 & 0 & 0 & 0 \\
0 & 0 & 1 & 0
\end{array}\right) \mathbf{x}_{k}+\mathbf{w}_{p o s, k}
$$


To include velocity measurements $\tilde{z}_{\text {velo, } k}$ measured in direction of movement the following equation

$$
\tilde{z}_{v e l o, k}=\sqrt{v_{x, k}^{2}+v_{y, k}^{2}}+w_{v e l o, k}
$$

has to be linearized around the current velocity estimation. Calculation of the Jacobian matrix results in

$$
\mathbf{H}_{k}=\left(\begin{array}{llll}
0 & h_{1} & 0 & h_{2}
\end{array}\right)
$$

with

$$
\begin{aligned}
h_{1} & =\frac{\hat{v}_{x, k}}{\sqrt{\hat{v}_{x, k}^{2}+\hat{v}_{y, k}^{2}}} \\
h_{2} & =\frac{\hat{v}_{y, k}}{\sqrt{\hat{v}_{x, k}^{2}+\hat{v}_{y, k}^{2}}} .
\end{aligned}
$$

Both white Gaussian measurement noise sequences $w_{\text {velo }, k}$ and $\mathbf{w}_{\text {pos }, k}$ are independent of each other and of the process noise. Its covariances are

$$
\mathbf{R}_{p o s, k}=\left(\begin{array}{cc}
\sigma_{x, k}^{2} & 0 \\
0 & \sigma_{y, k}^{2}
\end{array}\right)
$$

and

$$
R_{\text {velo }, k}=\sigma_{\text {velo }, k}^{2}
$$

To improve the state estimation with road map information two main calculation steps are implemented. These steps are performed when the current position estimation has left the road map or when the estimated direction of movement does not point into the direction of the current road tangent.

Initially the curve parameter value $l_{k}$ with minimal Euclidian distance to the current position is calculated, evaluating cross product for planar case. The current state estimation on the road is set to $x_{k}=s_{x}\left(l_{k}\right)$ and $y_{k}=s_{y}\left(l_{k}\right)$. Moreover the direction of movement is rotated towards the tangent of the road map at the curve $\mathbf{s}\left(l_{k}\right)$. Altogether the mean of the estimated state is updated, while the covariance of the state remains unchanged.

\section{B. Model in Local Curve Parameter Frame}

Assuming linear motion in local curve coordinate frame the time update of the current state vector $\mathbf{x}_{k}=\left(l_{k} v_{k}\right)^{T}$ can be written analogously to Eqn. (19) with

$$
\mathbf{F}=\left(\begin{array}{cc}
1 & T \\
0 & 1
\end{array}\right) \text { and } \mathbf{G}=\left(\begin{array}{c}
\frac{T^{2}}{2} \\
T
\end{array}\right)
$$

To update the current curve length estimation with incoming position measurements $\tilde{\mathbf{z}}_{\text {pos }, k}$ a transformation of local curve length coordinates to global measurement coordinates has to be carried out. The mathematical relation between both coordinate frames is given by the natural Hermite spline curve as described in Section II. The observation equation can be written as

$$
\tilde{\mathbf{z}}_{p o s, k}=\left(\begin{array}{c}
s_{x}\left(l_{k}\right) \\
s_{y}\left(l_{k}\right)
\end{array}\right)+\mathbf{w}_{p o s, k} .
$$

Calculation the Jacobian matrix around the current estimation on the road $l_{k}$ results in

$$
\mathbf{H}_{k}=\left(\begin{array}{ll}
h_{1} & 0 \\
h_{2} & 0
\end{array}\right)
$$

with

$$
\begin{aligned}
& h_{1}=b_{x, i}+2 c_{x, i}\left(\hat{l}_{k}-l_{i}\right)+3 d_{x, i}\left(\hat{l}_{k}-l_{i}\right)^{2} \\
& h_{2}=b_{y, i}+2 c_{y, i}\left(\hat{l}_{k}-l_{i}\right)+3 d_{y, i}\left(\hat{l}_{k}-l_{i}\right)^{2}
\end{aligned}
$$

for $\hat{l}_{k} \epsilon\left[l_{i}, l_{i+1}\right]$.

The observation equation to update the state estimation with incoming velocity measurements in direction of movement can be written as

$$
\tilde{z}_{v e l o, k}=\left(\begin{array}{ll}
0 & 1
\end{array}\right) \mathbf{x}_{k}+w_{v e l o, k}
$$

Again the error sources for measurement uncertainty are assumed to be uncorrelated and white, zero-mean distributed Gaussian random sequences. The measurement covariances are equal to the definition in Section IV-A.

While the measurement- and the time-updates are calculated the estimation $l_{k}$ remains on the road map and the direction of movement is exactly adjusted in direction of the road map tangent. Therefore a map matching as described in Section IV-A is only necessary to initialize the estimation process with the first incoming position measurement.

\section{NUMERICAL SIMULATIONS}

The performance of the developed object tracking algorithms was assessed in numerical simulations. Therefore both models were implemented in a framework to test modern rail localization techniques.

The covariances of the measurement noise are assumed to be given by the measurement concept, while the process noise is a design parameter that influences the performance of the tracking filter. Throughout this contribution both are set to fixed values.

To achieve realistic results, train characteristics are taken into account. Therefore accelerations remain within an interval of $-1.5 \mathrm{~m} / \mathrm{s}^{2} \leq a \leq 1.3 \mathrm{~m} / \mathrm{s}^{2}$ while velocities stay smaller than $25 \mathrm{~m} / \mathrm{s}$. Curvature values are chosen according to real rail tracks of secondary lines, with a minimum radius of around $155 \mathrm{~m}$.

The all over length of the test track is approximately $10470 \mathrm{~m}$. With one supporting point per $30 \mathrm{~m}$ the mean parameterization error as presented in section II decreases to $\bar{\epsilon}=1.62 \cdot 10^{-4} \mathrm{~m}$.

Initially, the true run of the track (see Figure 3) and velocities are integrated to obtain the object state, which is later used as a reference to calculate the errors of the estimated tracking solutions of both tracking strategies. Afterwards GPS positions [11] and velocities from an eddy current sensor system [12] are generated based on the true reference taken into account typical sensor error characteristics. 


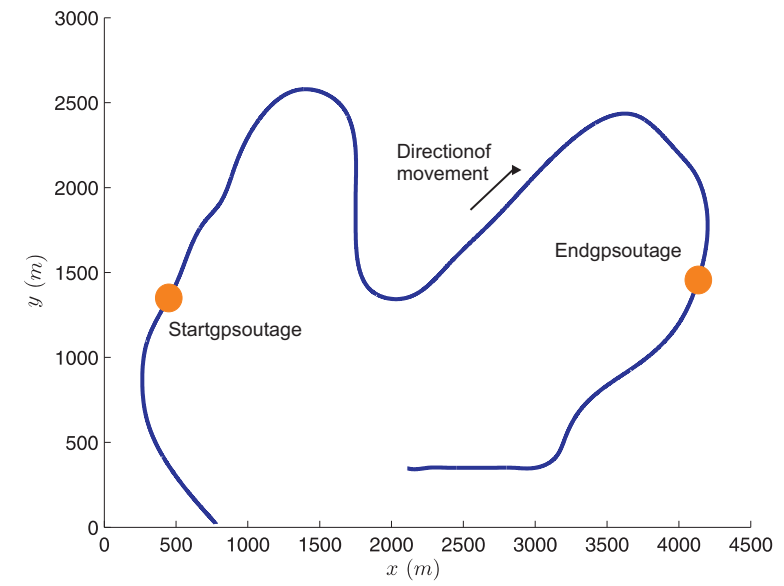

Fig. 3. Simulation test track including area of GPS outage used in simulation described in Section V-B

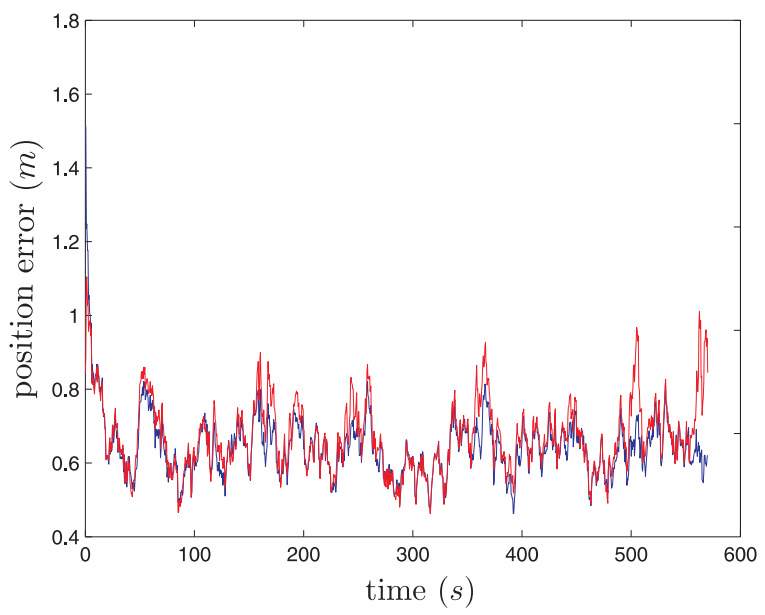

Fig. 4. Mean position accuracy of 50 numerical simulations with GPS position information available throughout the whole time interval for local curve model (blue) and global Cartesian model (red)

\section{A. Simulation with GPS available}

Throughout this simulation both, position and velocity measurement information, is available during the whole simulation interval of 600 seconds. The resulting position errors are depicted in Figure 4.

However both object tracking concepts offer quite good results in position accuracy.

\section{B. Simulation with GPS outage}

During this simulation a GPS outage is assumed starting at 100 second point and lasting for 300 seconds. During this time interval the tracking algorithms are only supported with map information and velocity measurements from the eddy current sensor system. The resulting position errors are depicted in Figure 5 and Figure 6.

In the case of a GPS outage the state space model in curve coordinates outperforms classical modelling in ground coordinates.

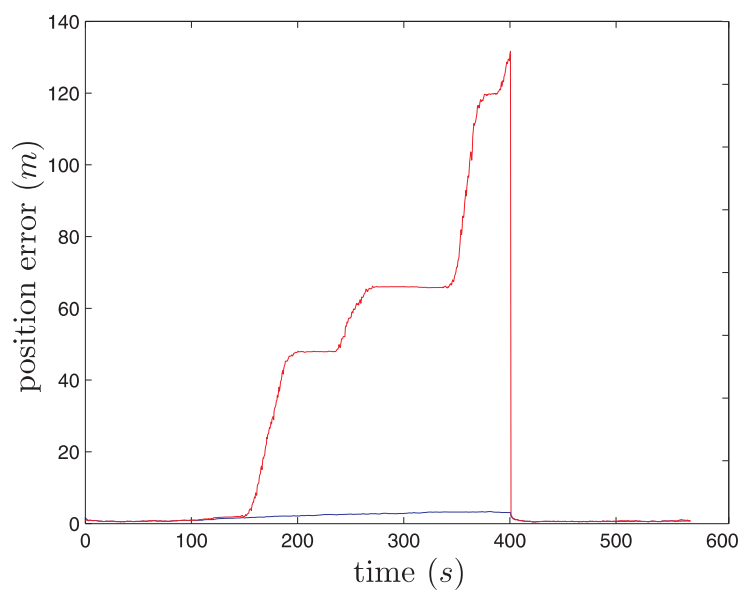

Fig. 5. Mean position accuracy of 50 numerical simulations with GPS outage of 300 seconds for local curve model (blue) and global Cartesian model (red)

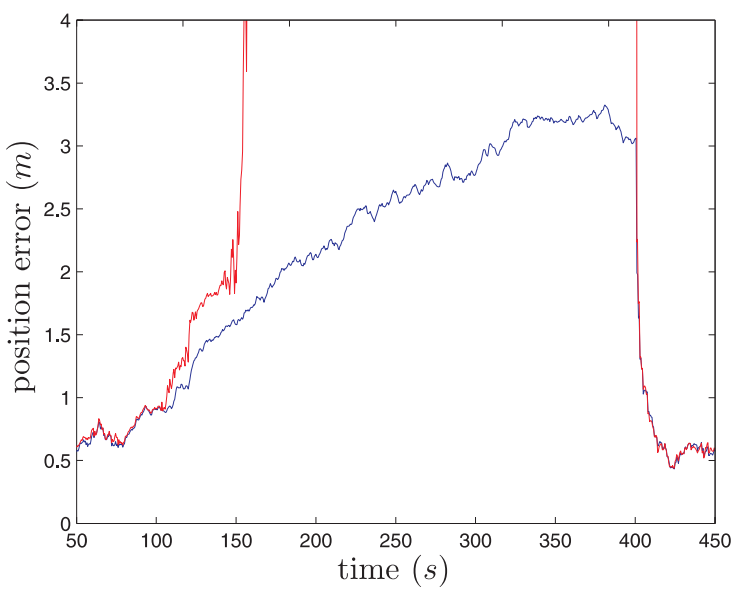

Fig. 6. Detail of the simulation results presented in Figure 5 with GPS outage of 300 seconds

\section{SUMMARY AND OUTLOOK}

Throughout this paper a geometric model based on Hermite spline curves to store road map information is presented, that can easily be modified for different tracking scenarios with varying accuracy requirements. Especially in case of strong curved roads or tracks the interpolation error remains significantly smaller than classical polygonal models.

To include these piecewise polynomial curves in object tracking algorithms arc-length parameterization has to be computed. Therefore a cost minimizing method is proposed to achieve good approximation results.

Two different approaches to combine this road map information source with additional sensor measurements are presented. While classical modelling in Cartesian coordinates yields an object that can reach every position in the plane measurement space, a definition in local curve coordinates results in an object that only can follow given road map elements. Assuming the road map to be known, the local 
curve model summarizes the past history of the system sufficiently and predicts future positions more precise. While time- and measurement updates are calculated the state estimation remains on the road constraint, a post filter step to project the estimation on the map is not necessary any more.

Both tracking algorithms are integrated in a framework to develop and validate modern train localization concepts. The simulation parameters are chosen according to train and track characteristics of secondary lines to achieve significant results. While absolute position measurements are available both tracking strategies offer similar performances. In case of lacking absolute position information the position accuracy of local curve modelling remains good while the position error of the second model rises on.

In future work a focus will be on advanced nonlinear filter methods, such as unscented Kalman filter or particle filter approaches to minimize linearization errors, which occur while the nonlinear state-to-measurement relations are evaluated.

\section{REFERENCES}

[1] Y. Bar-Shalom and T. Fortmann, Tracking and Data Association. Academic Press, 1988. isbn $=0120797607$.

[2] T. Kirubarajan, Y. Bar-Shalom, K. R. Pattipati, and I. Kadar, "Ground target tracking with variable structure imm estimator," IEEE Transactions on Aerospace and Electric Systems, vol. 36, pp. 26-46, 2000.

[3] M. Ulmke and W. Koch, "Road-map assisted ground moving target tracking," IEEE Transactions on Aerospace and Electric Systems, vol. 42, pp. 1264-1274, 2006.

[4] B. Pannetier, K. Benameur, V. Nimier, and M.Rombaut, "Vs-imm using road map information for a ground target tracking," in International Conference on Information Fusion (FUSION), 2005.

[5] C. Wenk, R. Salas, and D. Pfoser, "Adressing the need for mapmatching speed: Localizing global curve-matching algorithms," in 18th International Conference on Scientific and Statistical Database Managment, 2006.

[6] C. Yang, M. Bakich, and E. Blasch, "Nonlinear constrained tracking of targets on roads," in International Conference on Information Fusion (FUSION), 2005.

[7] G. Farin, Curves and Surfaces for CAGD. Morgan Kaufmann, 2002.

[8] M. S. Floater and T. Surazhsky, Topics in Multivariate Approximation and Interpolation, vol. ee, ch. Parameterization for curve interpolation, pp. 101-115. Elsevier B. V., 2005.

[9] P. S. Maybeck, Stochastic Models, Estimation and Control. Bd. 1, vol. 141 of Mathematics in Science and Engineering. New York: Academic Press, 1979. ISBN-10: 012480702X ISBN-13: 9780124807020 .

[10] R. Kalman and R. Bucy, "New results in linear filtering and prediction theory," in Transactions of the ASME - Journal of Basic Engineering, vol. 83, pp. 95-107, 1961.

[11] M. Grewal, L. Weill, and A. Andrews, Global Positioning Systems, Inertial Navigation and Integration. New York: John Wiley \& Sons, 2001. ISBN-10: 047135032X ISBN-13: 978-0471350323.

[12] T. Engelberg and F. Mesch, "Eddy current sensor system for noncontact speed and distance measurement of rail vehicles," in Computers in Railways VII (J. Allan, R. Hill, C. Brebbia, G. Sciutto, and S. Sone, eds.), (Southampton), pp. 1261-1270, WIT Press, 2000. 